Cahiers de recherches médiévales

La réception d'Isidore de Séville durant le Moyen Âge tardif $\left(X I I^{e}-X V^{e}\right.$ s.)

\title{
Dans le sillage d'Isidore de Séville
}

Le Tractatus de naturis animalium d'Engelbert d'Admont (ca 1250-1331)

\section{Max Schmitz}

\section{(2) OpenEdition \\ Journals}

Édition électronique

URL : https://journals.openedition.org/crm/10802

DOI : $10.4000 / \mathrm{crm} .10802$

ISSN : 1955-2424

Éditeur

Honoré Champion

Édition imprimée

Date de publication : 10 décembre 2008

Pagination : 175-194

ISSN : 1272-9752

Référence électronique

Max Schmitz, «Dans le sillage d'Isidore de Séville », Cahiers de recherches médiévales [En ligne], 16 |

2008, mis en ligne le 15 décembre 2011, consulté le 15 décembre 2022. URL : http://

journals.openedition.org/crm/10802; DOI : https://doi.org/10.4000/crm.10802 


\section{息 RM}

\section{Dans le sillage d'Isidore de Séville : Le Tractatus de naturis animalium d'Engelbert d'Admont (ca. 1250-1331)}

Abstract: Engelbert, abbot of Admont (ca. 1250-1331), is the author of a large number of works covering a wide range of subjects. Amongst them is an interesting text about the nature of creatures, the Tractatus de naturis animalium. Deeply inspired by the Etymologies of Isidore of Sevilla, Engelbert comments in this text different properties of the human nature and describes 243 animals in detail. Despite the existence of $13^{\text {th }}$ century encyclopaedias, which largely deal with these topics of the natural world, he prefers the six hundred years old Origines, as his model to follow. He keeps not only the same general structure, but he chooses more or less the same animals, provides the same type of information and cites the same sources. Surprisingly, almost every contemporary author is ignored. What Engelbert focuses on especially, is a careful selection and reformulation of the information given by his sources in order to make his own text as understandable as possible for a broad public.

Résumé: L'abbé Engelbert d'Admont (ca. 1250-1331), auteur de nombreuses ouvres touchant des sujets très divers, a également transmis à la postérité un texte intéressant sur la nature des créatures, le Tractatus de naturis animalium. S'appuyant largement sur les Etymologies d'Isidore de Séville, il commente une série de propriétés humaines et fournit la description de 243 animaux. Malgré l'existence d'encyclopédies quasi contemporaines couvrant ces aspects du monde naturel, les Origines, vieilles de 600 ans, sont pour lui le modèle à suivre, à la fois en ce qui concerne la structure, le choix des animaux, le type d'information à communiquer et les sources citées. Tout auteur contemporain est de facto passé sous silence. Loin d'être un simple compilateur, Engelbert reformule avec soin, sélectionne judicieusement ses informations et veille avec minutie à une compréhension aisée pour un public non averti.

Les œuvres d'Isidore de Séville ont été abondamment copiées tout au long du Moyen-Âge, et l'on en trouve des manuscrits dans toutes les grandes bibliothèques. Si son succès au Moyen-Âge ne fait aucun doute, l'utilisation de ses œuvres est beaucoup moins connue. Qu'en est-il de la réception de ses Étymologies aux derniers siècles de la période médiévale? Ce travail se propose d'éclairer un ouvrage inédit qui est fortement redevable des Origines, le Tractatus de naturis animalium ${ }^{1}$.

${ }^{1}$ Cet article synthétise le travail approfondi présenté en fin de cycle : Max Schmitz, Engelbert d'Admont (ca. 1250-1331) et son Tractatus de naturis animalium, mémoire de licence, Louvain-la-Neuve, Université catholique de Louvain, 2007. Il nous est agréable de remercier vivement ici M. Johann Tomaschek, le bibliothécaire de l'abbaye bénédictine d'Admont et M. Baudouin Van den Abeele, notre promoteur, pour leur aide précieuse et leurs conseils.

Cahiers de Recherches Médiévales, 16, 2008 


\section{Vie et œuvre d'Engelbert ${ }^{2}$}

Né en Styrie vers l'an 1250, Engelbert d'Admont reçoit sa première formation au monastère d'Admont (OSB) où il fait profession vers 1267. Dans une lettre $^{3}$ adressée à maître Ulric de Vienne ${ }^{4}$, Engelbert retrace lui-même différentes étapes de sa vie. Élève des maîtres Oscunus et Bohemil il étudie la logique et la grammaire en 1271 à l'école cathédrale de Saint-Guy à Prague. C'est Grégoire Zajic de Hasenburg, l'écolâtre de la cathédrale, qui lui fait découvrir les livres d'Aristote traitant de la nature. Suite à l'élection de Rodolphe I d'Habsbourg ( $1^{\mathrm{er}}$ octobre 1273) en tant que roi des Romains, des troubles politiques émergent et sur ordre d'Ottokar II Premysl, roi de Bohême ( $† 1278)$, les étudiants autrichiens et styriens sont évincés de Prague pendant l'hiver 1274-1275. De retour à Admont, Engelbert écrit son premier ouvrage De electione regis Rudolphi et peut en 1276 offrir au roi la première partie de cette oeuvre.

Peu après, Engelbert quitte à nouveau Admont et part pour Padoue avec un

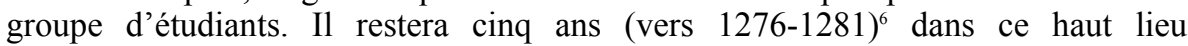
universitaire imprégné fortement par l'aristotélisme et partiellement par l'averroïsme ${ }^{7}$. Parmi ses maitres, figure Guillaume de Brescia (Guillaume de Corvi, $\dagger$ 1326), professeur de logique et de philosophie, lui-même élève de Taddeo Alderotti (1223-1295). Après le départ de Guillaume de Brescia, Engelbert étudie la théologie au monastère dominicain de St-Augustin de Padoue (1281-1285). Engelbert copie des extraits des œuvres aristotéliciennes, ramène ses manuscrits en Styrie $^{8}$ et introduit ainsi les idées du grand penseur en Autriche.

\footnotetext{
${ }^{2}$ Pour des informations détaillées concernant la vie d'Engelbert, voir M. Hamm, « Engelbert von Admont», dans Die deutsche Literatur des Mittelalters. Verfasserlexikon, t. 2, BerlinNew York, 1980, col. 535-549. P. Ernstbrunner, Der Musiktraktat des Engelbert von Admont (ca. 1250-1331), Tutzing, 1997. K. Ubl, Engelbert von Admont. Ein Gelehrter im Spannungsfeld von Aristotelismus und christlicher Überlieferung, Vienne-Munich, 2000, p. 13.

${ }^{3}$ La lettre à Ulric a été écrite vers 1325. Elle est éditée dans G. B. Fowler, «Letter of Abbot Engelbert of Admont to master Ulrich of Vienna», Recherches de théologie ancienne et médiévale, 29, 1962, p. 298-306.

${ }^{4}$ Voir F. J. Worstbrock, «Ulrich von Wien», dans Die deutsche Literatur des Mittelalters. Verfasserlexikon, t. 10, Berlin-New York, 1996, col. 53-55. F. P. Knapp, «Leben und Werk des Magisters Ulrich von Wien. Eine vorläufige Skizze », dans Ir sult sprechen willkommen. Festschrift für Helmuth Birkhan zum 60. Geburtstag, Berne, 1998, p. 788-797.

${ }^{5}$ Pia Ernstbrunner, Der Musiktraktat des Engelbert von Admont (ca. 1250-1331), Dissertation zur Erlangung des Doktorgrades der Philosophie, Vienne, Geisteswissenschaftliche Fakultät der Universität Wien, 1995, p. 7.

${ }^{6}$ La date exacte de son arrivée à Padoue fait l'objet de discussions. Voir K. Ubl., «Zur Entstehung der Fürstenspiegel Engelberts von Admont (†1331)», Deutsches Archiv für Erforschung des Mittelalters, 55, 1999, p. 508-509. P. Ernstbrunner, op. cit., p. 8-10.

${ }^{7}$ F. Kucher, «Der Bildungsgang und das philosophische Umfeld Engelberts von Admont», dans W. Baum (sous la dir.), Engelbert von Admont. Vom Ursprung und Ende des Reiches und andere Schriften, Graz, 1998, p. 227.

${ }^{8}$ Un de ces manuscrits est sans doute le Codex Admontensis 608. F. Kucher, op. cit., p. 234.
} 
De retour en Styrie, Engelbert est nommé à la tête de l'abbaye St-Pierre à Salzbourg où il restera pendant onze ans (1286-1297) . Suite à l'assassinat de l'abbé d'Admont Henri II, Engelbert est élu abbé de ce monastère en juin $1297^{10}$. Parmi ses amis, figurent l'archevêque Conrad IV de Salzbourg (†1312), l'abbé Jean de Viktring († 1345/1347) et le maître Ulric de Vienne ${ }^{11}$. Engelbert préside l'abbaye jusqu'à sa résignation en $1327^{12}$. Il meurt en mai 1331 .

Auteur prolifique, Engelbert a cité les titres de la majorité de ses œuvres dans sa lettre à Ulric. Il fait état de 33 œuvres dont 16 se rapportent à la théologie, 8 à la philosophie naturelle et 9 à la philosophie morale. Les titres des oeuvres de philosophie naturelle, qui nous intéressent le plus dans ce contexte, sont les suivants: 1) Commentarius magnus super librum Aristotelis de mundo ; 2) Commentarius sive scriptum super librum Aristotelis de inundatione Nili ; 3) Tractatus de fascinacione; 4) Tractatus de naturis animalium; 5) Tractatus de quibusdam naturalibus; 6) Tractatus de causis et signis mutationis aeris et temporum ; 7) Tractatus de causis longevitatis hominum ante diluvium et 8) Tractatus de musica ${ }^{13}$.

Outre les 33 œuvres mentionnées plus haut il en existe au moins encore six autres que l'on peut qualifier d'authentiques ${ }^{14}$. Est encore à ajouter la lettre adressée à Ulric et celle à Conrad IV de Salzbourg, cette dernière étant la nouvelle préface au commentaire du Psaume $118^{15}$. Trois œuvres attribuées à Engelbert sont douteuses ${ }^{16}$ et trois autres ne sont définitivement pas de sa plume ${ }^{17}$. De ces 44 œuvres, il existe environ 213 manuscrits ${ }^{18}$ qui les contiennent ou les contenaient ${ }^{19}$.

\footnotetext{
${ }^{9}$ K. Ubl, art. cit., p. 502-505.

${ }^{10}$ Engelbert émet sa première charte comme abbé d'Admont le 24 septembre 1297 à Vienne. Die Regesten der Erzbischöfe und des Domkapitels von Salzburg. 1247-1343, t. 2, éd. F. Martin, Salzbourg, 1931, n 323, p. 40 et n 346, p. 43.

${ }^{11}$ Voir W. Stelzer, "Gebildete Zeitgenossen im Umkreis Engelberts von Admont», dans J. Tomaschek et M. Braunsteiner (sous la dir.), Abt Engelbert von Admont (reg. 1297-1327), Admont, 1998, p. 18-35.

${ }^{12}$ La raison qu'il invoque pour sa démission est l'âge et le désir de se consacrer entièrement à l'écriture de ses livres, ce qui lui sera possible une fois libéré des devoirs quotidiens: [...] propter senectutem et fragilitatem corpoream, ut a curis temporalibus absolutus quieti contemplacioni vacare et scribendis libris sollicius operam dare possit [...]. F. M. Mayer, «Beiträge zur Geschichte des Erzbistums Salzburg. 2 : Über ein Formelbuch aus der Zeit des Erzbischofs Friedrich III », Archiv für österreichische Geschichte, LXII, 1881, p. 190.

${ }^{13}$ Les œuvres 1, 2, 5 et 6 ne sont probablement pas conservées. Les œuvres 3, 7 et 8 sont éditées. Nous avons fait une première édition de la 4 e ouvre. M. Schmitz, op. cit., p. 262-350.

${ }^{14}$ 1) Meditacio et recapitulatio articulorum passionis domini ; 2) Questiones de sanctissima trinitate ; 3) Psalteria ; 4) Tractatus de officio ancillari B.M.V. ; 5) Tractatus de passione domini secundum Matthaeum et 6) Libellus de XII questionibus specialibus de rebus ad fidem spectantibus. Voir K. Ubl, op. cit., p. 23.

${ }^{15}$ Voir G. B. Fowler, «A new dedicatory preface to the commentary on Ps. 118 by Engelbert of Admont », Recherches de théologie ancienne et médiévale, 29, 1962, p. 306-312.

${ }^{16}$ De animabus; Metra sequentia de intervallo et Questio super illo verbo quod cantat ecclesia in die resurrectionis : Tu factus es spes desperatis.

${ }^{17}$ Les titres des oeuvres sont: Speculum militare; Tabulae quaedam cum canonibus et perspectiva et Sermones de tempore et de sanctis. Voir F. Kucher, « Handschriftenverzeichnis
} 
Le Tractatus de naturis animalium

Le traité d'Engelbert portant sur les êtres animés est conservé dans trois manuscrits du XIV siècle. On connaissait jusqu'il y a peu deux manuscrits seulement, tous deux à $\mathrm{Admont}^{20}$, mais il en existe un troisième manuscrit conservé à Ljubljana ${ }^{21}$. Le manuscrit Ljubljana 23 et le Cod. Adm. 119 sont les témoins les plus anciens et présentent la meilleure qualité textuelle. Le manuscrit Cod. Adm. 547 semble être une copie directe du Cod. Adm. 119.

\section{Structure}

Le traité d'Engelbert portant sur les êtres animés se compose d'un prologue et de deux parties principales de taille inégale. La première, plus courte, est consacrée à l'homme et aux races monstrueuses; la deuxième, plus importante, aux animaux au sens strict. M. Köhler ayant analysé surtout la première partie ${ }^{22}$, notre intérêt porte plus sur la deuxième.

der Werke Engelberts von Admont », dans W. Baum (sous la dir.), op. cit, p. 276-300 [liste fautive !]. K. Ubl et A. Sauter, «Johann von Viktring als Autor des Speculum militare», Deutsches Archiv für Erforschung des Mittelalters, 57, 2001, p. 515-553. K. Ubl, op. cit., p. 21, note de bas de page 71. M. Burckhardt et G. Meyer, Die mittelalterlichen Handschriften der Universitätsbibliothek Basel. Beschreibendes Verzeichnis. Abteilung B: Theologische Pergamenthandschriften, t. 2: Signaturen B VIII 11 - B XI 26, Bâle, 1966, p. 383-384. Au sujet de cet auteur voir B. M. Von Scarpatetti, Die Handschriften der Stiftsbibliothek St. Gallen. Beschreibendes Verzeichnis. Codices 1726-1984, Saint-Gall, 1983, p. 243-244. Les sermons contenus dans le manuscrit Innsbruck, Universitätsbibliothek, Cod. 468, f. 197ra-198vb ne sont pas d'Engelbert d'Admont mais d'Engelbert de Cologne (OM).

${ }^{18}$ L'oeuvre De passione beate Katherine n'est de fait pas contenue dans les trois manuscrits cités par Kucher. G. Glauche, Katalog der lateinischen Handschriften der Bayerischen Staatsbibliothek München: Die Pergamenthandschriften aus dem Domkapitel Freising, t. 1 : Clm 6201-6316, Wiesbaden, 2000, p. 114.

${ }^{19}$ Nous en avons dressé l'inventaire. M. Schmitz, op. cit., p. 53 et annexes n ${ }^{\circ} 10$ et 11.

${ }^{20}$ Admont, Stiftsbibliothek, Cod. Adm. 119, f. 29r-47r et Admont, Stiftsbibliothek, Cod. Adm. 547, f. 82r-120v.

${ }^{21}$ Le manuscrit a été découvert par Baudouin Van den Abeele. Ljubljana, Narodna in Univerzitetna Knjiznica, Ms 23, f. 43v-73r. Voir http ://www.ksbm.oeaw.ac.at/kataloge/SI/SI3000/SI3000-23.pdf; consulté le 15 juin 2008.

${ }_{22}$ T. W. Köhler, «De natura hominis - Engelbert von Admont und das philosophische Erkenntnisbemühen um den Menschen im 13. Jahrhundert», dans J. Tomaschek et M. Braunsteiner, op. cit., p. 58-77. 
Tableau de la structure et du contenu du De naturis animalium ${ }^{23}$

\begin{tabular}{|l|r|r|}
\hline Prologue & & $29 \mathrm{ra}$ \\
I.1) De la nature humaine & 21 chapitres & \\
I.2.1) Des genres monstrueux d'hommes & & $39 \mathrm{ra}-30 \mathrm{vb}$ \\
I.2.2) Des genres de monstres & & $30 \mathrm{vb}-31 \mathrm{ra}$ \\
& & $31 \mathrm{ra}-31 \mathrm{va}$ \\
II.1.1) De la nature des animaux : & \\
$\quad$ Définition de l'animal & & $31 \mathrm{va}$ \\
II.1.2) Division de l'animal & & $31 \mathrm{va}$ \\
II.1.3) Deux questions & & $31 \mathrm{va}$ \\
II.1.3.1) Réponse à la première question & & $31 \mathrm{vb}$ \\
II.1.3.2) Réponse à la deuxième question & & $31 \mathrm{vb}$ \\
II.1.4) Des genres d'animaux & & $31 \mathrm{vb}$ \\
& & \\
II.2.1) Des animaux marchant sur terre & 72 chapitres & $32 \mathrm{ra}-38 \mathrm{va}$ \\
II.2.2) Des serpents & 28 chapitres & $38 \mathrm{va}-40 \mathrm{ra}$ \\
II.2.3) Des vers & 11 chapitres & $40 \mathrm{ra}-40 \mathrm{va}$ \\
II.2.4) Des poissons & 53 chapitres & $40 \mathrm{va}-42 \mathrm{vb}$ \\
II.2.5) Des oiseaux & 68 chapitres & $43 \mathrm{ra}-46 \mathrm{vb}$ \\
II.2.6) Des petits animaux volants & 18 chapitres & $46 \mathrm{vb}-47 \mathrm{rb}$ \\
\hline
\end{tabular}

Pour la première partie (de natura hominis), M. Köhler propose deux subdivisions, la première étant consacrée à la création, la composition et les propriétés du corps humain, la deuxième aux différentes formes de l'être humain. En effet, l'homme peut différer selon l'âge, le tempérament ou le sexe. Cette subdivision comprend également les différents types d'hommes déformés comme les géants, centaures etc. M. Köhler insiste sur le fait qu'Engelbert n'y traite à aucun moment de l'âme humaine. La structure de cette partie est copiée en partie sur la composition du livre XI des Étymologies d'Isidore de Séville ${ }^{24}$.

Dans la partie consacrée aux animaux, le traité d'Engelbert adopte manifestement la structure établie par Isidore dans ses Origines ${ }^{25}$. Tout comme Isidore, Engelbert distingue trois grandes sections selon le milieu de vie: animaux de terre, d'eau et d'air. Isidore ventile ce classement en huit catégories ${ }^{26}$, système

\footnotetext{
${ }^{23}$ L'édition du texte se base essentiellement sur le Cod. Adm. 119. Les folios de ce manuscrit sont indiqués dans la colonne à droite. C'est chaque fois au texte édité que nous renvoyons dans ce travail. La numérotation des chapitres tient compte de la structure du texte et a été ajoutée par nos soins.

${ }^{24}$ T. W. Köhler, art. cit., dans J. Tomaschek et M. Braunsteiner, op. cit., p. 62-70.

${ }^{25}$ Isidore de Séville, Étymologies, livre XII : Des animaux, éd. et trad. J. André, Paris, 1986. The Étymologies of Isidore of Seville, trad. S. A. Barney (et autres), Cambridge, 2006, p. 24.

${ }^{26}$ Pour ses huit catégories Isidore s'est inspiré chez Pline l'Ancien qui distingue quatre catégories : animaux quadrupèdes domestiques et sauvages (livre VIII), animaux aquatiques
} 
qu'Engelbert garde implicitement. Bien qu'il regroupe les trois premières catégories d'Isidore dans une seule, sous le nom de De gressibilibus, la deuxième et troisième catégorie d'Isidore (De bestiis, De minutis animantibus) se retrouvent toujours à l'intérieur de celle-là. Les cinq autres catégories (de serpentibus, de vermibus, de piscibus, de avibus, de minutis volatilibus) sont reprises telles quelles ${ }^{27}$.

Contrairement à ce qui s'observe dans le texte d'Isidore, chacune des six catégories débute par une introduction nettement délimitée, qui fournit des informations générales sur les animaux en question. Engelbert avance l'argument de notoriété pour justifier sa procédure de traiter d'abord des animaux se déplaçant sur terre, puis des animaux aquatiques et enfin des animaux volants ${ }^{28}$. Ce même argument vaut également au sein de la catégorie de gressibilibus pour commencer par les animaux domestiques ${ }^{29}$.

Comme il ne suit pas d'ordre alphabétique, Isidore a procédé à des regroupements pour garder une certaine logique dans son classement des huit catégories. Ces rassemblements sont sans césure visuelle, sans sous-titrages. Engelbert fait de même.

Chez les deux auteurs, le groupement et le classement des animaux reposent sur des critères variables ${ }^{30}$ : initiale du nom de l'animal ${ }^{31}$, ordre d'apparition de ces animaux dans la source ${ }^{32}$ et similarités morphologiques ${ }^{33}$, comportementales $^{34}$ ou d'espace vital ${ }^{35}$.

À l'intérieur de la catégorie des animaux marchants, Engelbert commence tout comme Isidore par les animaux domestiques (de bove à de asino), continue avec les animaux sauvages (de onagro à de tragelapho), puis passe aux animaux dangereux (de leone à de furone) et petits animaux (de muribus à de ericio). À la suite des petits animaux, il ajoute une sous-catégorie composée d'animaux non décrits par Isidore, mais qui proviennent tous des Collectanea rerum memorabilium de Solin $\left(3^{\mathrm{e}}-4^{\mathrm{e}}\right.$ siècle) (de wisonta à de eale). Ces animaux sont presque tous dangereux et vivent dans des régions lointaines (Inde, Afrique), à l'exception du

(livre IX), oiseaux (livre X) et insectes (livre XI) et chez Aristote notamment pour ses critères de distinction. Isidore de Séville, op. cit., p. 8.

${ }^{27}$ Il n'y a que deux ou trois inversions dans ces cinq catégories. Voir les notices de thynnis [II, $2,4,7]$ et de ficedula [II, 2, 5, 59].

${ }^{28}$ Ordo autem hic, ut superius $<$ di $>$ ctum est secundum nociora est, quia terrestria nociora nobis sunt primo loco; deinde aquatilia; ultimo volatilia. Sermo generalis de avibus [II, 2, $5,1]$.

${ }^{29}$ Primo ergo de domesticis, que ex usu nociora sunt, dicamus propter legentis solacium magis quam ad profectum discencium. De gressibilibus [II, 2, 1, 1].

${ }^{30}$ Isidore de Séville, op. cit., p. 8.

${ }^{31}$ Voir par exemple mullus et mugilis. Ibidem, 6, 25-26, p. 196-197. Engelbert d'Admont, Tractatus de naturis animalium, [II, 2, 4, 21-22].

${ }^{32}$ Voir par exemple draco marinus et murena. Isidore de Séville, op. cit., 6, 42-43, p. 206-207 et Pline l'Ancien, Historia naturalis, 32, 14. Voir aussi leocrota et eale. Solin, Collectanea rerum mirabilium, LII, 34-35. Engelbert d'Admont, op. cit., [II, 2, 1, 70-71].

${ }^{33}$ Voir grifes, pegasus et tragopan. Engelbert d'Admont, op. cit., [II, 2, 5, 64-66].

${ }^{34}$ Voir par exemple les animaux carnivores. Isidore de Séville, op. cit., 2, 3-11, p. 86-95.

${ }^{35}$ Voir par exemple ursus et ystrix. Engelbert d'Admont, op. cit., [II, 2, 1, 45-46]. 
bison (wisonta). Il se peut qu'en les plaçant ainsi, Engelbert ait eu le souci de ne pas perturber l'arrangement d'Isidore, alors qu'ils seraient plus à leur place dans la catégorie des animaux non domestiqués ou bêtes féroces (sauf pour le phalangium).

Engelbert a repris presque tous les animaux qui se trouvent dans le douzième livre d'Isidore ${ }^{36}$. Même s'il a fusionné plusieurs notices dans une seule ${ }^{37}$ ou s'il a déplacé une notice ailleurs, tous les animaux sont intégrés à l'exception du poney (mannus), du deuxième serpent seps et des autres espèces de serpents (ammoditae, elephantiae, camaedracontes) évoqués par Isidore.

En général Engelbert décrit les animaux selon l'ordre d'Isidore, et ce n'est que dans la première catégorie qu'Engelbert procède à un véritable remaniement ${ }^{38}$. De même, à l'intérieur d'un chapitre, Engelbert respecte plus ou moins la structure du texte de ses sources.

Au catalogue des animaux d'Isidore, l'abbé d'Admont ajoute 12 animaux terrestres (de wisonta à de eale) [II, 2, 1, 60-71] et 4 oiseaux (strophylos, caristee aves, pegasus et tragopan) [II, 2, 5, 16, 63, 65 et 66] issus du texte de Solin. De même la première notice de la girafe [II, 2, 1, 17] (décrite en fait à deux reprises sous deux noms différents) peut être considérée comme l'emprunt à Solin d'un « nouvel » animal.

Dans ses 243 notices animalières traitées en détail Engelbert ne se limite pas à ces deux auteurs comme source d'inspiration. En effet, il a intégré trois autres notices d'auteurs non définis. À la notice sur l'écureuil (spariolus) ${ }^{39}$, Engelbert ne fournit aucune source, à celle du chapon (gallus gallinacius $)^{40}$, il renvoie à une source appelée lapidarius et à la troisième, celle du rossignol (phylomena) il évoque des physiologit $i^{41}$.

\footnotetext{
${ }^{36}$ Nous avons pris en considération pour Engelbert d'Admont tous les titres rubriqués du Cod. Adm. 119 dont le premier est de bove et pour Isidore de Séville le nom de l'animal qui figure en tête de chacun des paragraphes de l'édition de J. André.

${ }^{37}$ Voir la notice de solifuga [II, 2, 1, 52].

${ }^{38}$ Voir par exemple les notices de leone [II, 2, 1, 31] et de leopardo [II, 2, 1, 32].

${ }^{39}$ Spariolus a spargiendo dictus est, quia nuces et fructus, quibus vescitur hinc, inde spargit, donec eligat, quod melius est ex hiis et est animal parvum, habens capud solidum et forte et est acuti visus. Caudam habet magnam et pilosam, pilis subtilibus ad modum pennarum, unde et de arbore ad arborem volat et a pluvia se protegit, cauda superposita corpori a retro usque ad caput. Cum senescunt, pellis eorum incipit rubere parum. Quando non inveniunt sufficienciam et bonitatem pascue, per multas provincias et regiones, de silva ad silvam currunt gregatim, et duos custodes, unum ante se et alium post se, destinant. Fluvios pedum nisu et caude velo transnatant. Dentes binos habent acutissimos ad conterendum testas nucum vocem fortissimi soni emittunt valde horribilis. Alii eorum nidos habent in arboribus inferius, circa radices; alii in cuniculis et cavernis terre. De spariolo [II, 2, 1, 23].

${ }^{40}$ Gallus gallinacius est gallus castratus. In huius ventre post tres annos nascitur lapis preciosus, qui vocatur allectorium, ut dicitur in lapidario. Iste gallus non cantat, non generat, non ambulat cum gallina, nec curat, nec custodit eam, quia perdidit iactanciam, quam habebat in testiculis, sed nichil aliud facit, nisi quod multum comedit et facile inpingwatur et ideo ad nichil est utilis nisi ad coquinam. De gallo gallinacio [II, 2, 5, 38].

${ }^{41}$ Phylomena est avicula parva per estatem nocte dulcissime cantans, ita ut ova sua non minus fotu quam cantus swavitate fetificet. Unde dicunt physiologii quod per horam incubat
} 


\section{Ampleur des catégories chez Isidore et Engelbert}

Alors que les catégories ont proportionnellement la même taille chez Isidore et Engelbert, leur volume varie selon les auteurs. Engelbert, lui a tendance à amplifier ses chapitres animaliers ${ }^{42}$. Ainsi la plus grande catégorie, celle des animaux marchant sur terre, comprend chez Engelbert 11.471 mots, tandis que chez Isidore, où elle correspond au regroupement de ses trois premières catégories, cela ne fait que 4.081 mots. Viennent ensuite la catégorie des oiseaux (avec 7.723 mots chez Engelbert et 2.835 mots chez Isidore), celle des poissons (4.546 contre 1.925 mots), devant les serpents, les vers et les petits animaux volants, qui ne font que 810 mots chez Engelbert et 427 mots chez Isidore. La différence de volume est la moindre dans la partie sur les serpents (844 mots environ), ce qui fait un surplus de $48,3 \%$ de l'abbé par rapport à l'évêque. Ici Engelbert a inséré bien moins d'informations étrangères et s'est contenté le plus souvent de reprendre le texte d'Isidore.

L'accroissement du texte d'Engelbert par rapport à celui d'Isidore tient à deux facteurs. D'une part, Engelbert a souvent complété le texte des Étymologies par d'autres sources ${ }^{43}$ et d'autre part, le style d'Engelbert est moins concis que celui d'Isidore. En effet, Isidore emploie un style très concentré et bref, à la fois pour la construction des phrases et pour l'information transmise. On peut qualifier ce style de sententialis brevitas ${ }^{44}$. Isidore voulait sans doute par ce processus rendre le texte plus clair et simple au lecteur. Engelbert par contre, dans ce même souci de compréhension et de clarté, donne bien plus d'explications et fait usage d'un style plus long et plus répétitif ${ }^{45}$. Ainsi fait-il un ample usage d'adjectifs démonstratifs et d'adverbes. À titre d'exemple nous pouvons citer la notice du dail, une sorte de coquillage :

ovo et postea iterum dextro pede stans super ovum cantat. Est autem avicula multum veneri dedita et adeo delicatur suo cantu super memoriam veneris aut etiam ovorum fovendorum, quod rupto cerebro pre nimietate cantus moritur et inde dicitur phylomena a greco phylos, quod est amor et mene, quod est defectus, inde philomena quasi amore deficiens.

De phylomena [II, 2, 5, 56].

${ }^{42}$ L'accroissement dans les trois plus grandes catégories varie entre $+136,2$ et $181,1 \%$ des mots)

${ }^{43}$ Il faut quand même souligner qu'Engelbert laisse aussi de côté certains passages de sa source principale.

${ }^{44}$ J. Fontaine, Isidore de Séville et la culture classique dans l'Espagne wisigothique, t. 2, Paris, 1983, p. 768.

${ }^{45}$ Finally, the style of the tract (de officiis et abusionibus eorum) shows plainly certain established characteristics of Engelbert's writing, such as the love of repetition, the use of two words of almost the same import where most authors would be content with one, and the combination of scholastic and classical turns of phrase peculiar to him. G. B. Fowler, «Tractatus de officiis et abusionibus eorum », dans Essays in medieval Life and Thought, presented in honor of Austin Patterson Evans, New York, 1955, p. 111-112. 


\begin{tabular}{|l|l|}
\hline Isidore de Séville & Engelbert d'Admont \\
\hline $\begin{array}{l}\text { Ungues a similitudine humanarum unguium } \\
\text { dictae }^{46} .\end{array}$ & $\begin{array}{l}\text { Ungwes etiam quoddam genus conche } \\
\text { vocantur ita quia similitudinem humanorum } \\
\text { ungwium habent }\end{array}$ \\
\hline
\end{tabular}

\section{Problèmes de terminologie}

Par moments, Engelbert se voit confronté à des problèmes de terminologie. Ainsi, à quelques reprises il a rattaché deux noms à un même animal, alors que chez Isidore et en réalité il s'agit de deux animaux différents. C'est le cas du mus araneus: Engelbert est d'avis que mus araneus et solifuga désignent le même animal. Or mus araneus est le nom latin de la musaraigne et solifuga est une espèce d'araignée lucifuge ${ }^{48}$. Il a fait le même type d' «erreur» dans la description des bogues (de boccis), du serpent enhydris ou du bouquetin (de ybice). Dans les quatre cas, c'est la proximité dans le texte qui est probablement responsable de ces attributions de noms. Chaque fois, les notices se suivent directement et Engelbert n'a pas remarqué la césure entre les deux descriptions ; en d'autres mots, il a joint ce qui devait rester disjoint ${ }^{49}$.

Il arrive qu'Isidore rédige des notices utilisant deux fois le même nom d'animal. Ainsi décrit-il à deux reprises le serpent seps $(4,17$ et 31$)$ et le serpent dipsas (4, 13 et 32). Cette répétition pourrait résulter d'une faute de négligence. Engelbert a constaté qu'il y avait là matière à critique. Il résout le premier cas en laissant tomber tout simplement la description du deuxième serpent $\operatorname{seps}^{50}$. Dans le cas du serpent dipsas, il a consulté le texte de Solin et a conclu qu'il s'agit de deux serpents différents. Il précise dès lors qu'il est bien conscient qu'il vient tout juste de décrire un serpent du même nom ${ }^{51}$.

Dans la catégorie des petits animaux volants un problème analogue se présente. Isidore évoque les deux noms du faux-bourdon (costrus et fucus $)^{52}$ sans

\footnotetext{
${ }^{46}$ Isidore de Séville, op. cit., 6, 55, p. 216-217.

${ }^{47}$ De ungwibus [II, 2, 4, 49].

${ }^{48}$ Isidore de Séville, op. cit., 2, 4, p. 126-127.

${ }^{49}$ Ceci s'observe parfois aussi chez Isidore. Isidore de Séville, op. cit., p. 24.

${ }^{50}$ Dans sa description du prester, Engelbert suit de près le texte d'Isidore, or celui-ci a inséré dans sa notice du prester des éléments repris à Solin, qui appartiennent au seps. Voir les notices de prestero [II, 2, 2, 9] et de sepe [II, 2, 2, 10]. Isidore de Séville, op. cit., p. 24 et 4, 16-17, p. 144-145.

${ }^{51}$ Dypsa, ut dicunt Ysidorus et Solinus, est genus serpentis, que Latine situla dicitur. Hic si quem momorderit, pre siti morietur. De dypsa [II, 2, 2, 7].

Dypsa serpens quidam idem nomine, sed specie diversus ab eo, quem superius nominavimus, ut dicit Solinus et est tante exiguitatis, quod non videtur, dum calcatur et est etiam tanti veneni ut, antequam lesus exinde dolorem senciat, moriatur. Unde poeta : Signiferum iuvenem Tyrreni sangwinis $A<u l>$ um tort $<_{O}>$ caput retro dypses calcata momordit: vix dolor aut sensus dentis fuit et cetera [...]. De dyps $<a>$ [II, 2, 2, 24].

${ }_{52}$ Costros Graeci appellant qui in extremis fauorum partibus maiores creantur; quos aliqui reges putant. Dicti quod castra ducant.
} 
préciser si pour lui il s'agit du même animal ou de deux animaux différents. Engelbert quant à lui fait une nette distinction et affirme tel Virgile que le costrus de la «famille » des abeilles naît du cadavre de bœufs et le fucus de la putréfaction des mulets $^{53}$.

On constatera qu'Engelbert décrit deux fois la girafe ${ }^{54}$. Ainsi fait-il un chapitre portant le titre de pardalo (II, 2, 1, 17) et un deuxième qu'il intitule de cameleopardo (II, 2, 1, 39). Pour le premier, il se réfère à Solin (Collectanea rerum mirabilium, XXX, 19), tandis que pour le deuxième il s'inspire de la notice d'Isidore. Comme Isidore emploie un autre nom pour cet animal, Engelbert ne s'est pas aperçu que celui-ci s'est basé lui-même sur le texte de Solin. Vu que la girafe ne se trouve pas dans le regroupement isidorien des camélidés $(1,35$ et 36$)$, alors qu'elle a des points en commun avec le chameau, Engelbert a sans doute pensé qu'Isidore avait oublié cet animal et par conséquent il a «complété » le texte.

Ce qui frappe dans le traité d'Engelbert est sa volonté de clarifier des passages restés obscurs dans le texte d'Isidore et d'essayer de définir l'espèce des animaux. Ainsi, comme il n'a pas trouvé l'étymologie du nom de l'animal dans la notice isidorienne du bupreste, il a décidé de proposer lui-même une explication (assez fantaisiste) : Buprestis dicitur quasi boves premens. Dans la même notice, Engelbert précise explicitement, contrairement à Isidore, que cet animal est du genre volant (Est autem volatilis genus). Cette information est superflue puisque la catégorie à laquelle appartient cet animal est déjà celle des petits animaux volants. Un autre bon exemple est la notice du poisson uranoscope :

\begin{tabular}{|c|l|}
\hline \multicolumn{1}{|c|}{ Isidore de Séville } & \multicolumn{1}{|c|}{ Engelbert d'Admont } \\
\hline $\begin{array}{l}\text { Uranoscopus uocatur ab oculo, } \\
\text { quem in capite habet, a quo semper } \\
\text { supra intendit }{ }^{55} .\end{array}$ & $\begin{array}{l}\text { Uranoscopus, ut dicit Ysidorus, est } \\
\text { quidam piscis et vocatur a Greco uran, quod est } \\
\text { celum et scopos, quod est intendere, inde } \\
\text { uranoscopus quasi celum intendens. Habet enim } \\
\text { unum oculum et cum illo semper aspicit } \\
\text { sursum }\end{array}$ \\
\hline
\end{tabular}

Fucus est maior ape, scabrone minor. Dictus autem fucus quod alienos labores edat, quasi fagus; depascitur enim quod non laborauit. De quo Vergilius: Ignauum fucus pecus a praesepibus arcet. Isidore de Séville, op. cit., 8, 3, p. 286-289.

${ }_{53}$ Virgilius in IIII ${ }^{\text {to }}$ Georgicis dicit quod artificialiter possent generari apes de putrefactis carnibus boum vel vitulorum, sicut scabrones de equis. Fuci de mulis vespe de asinis. De apibus [II, 2, 6, 2].

${ }^{54}$ Solinus refert quod in Ethyopia est animal, quod apud illos vocatur nabus, apud nos camelus pardalus dicitur et habet collum simile collo equi et pedes, sicut bubalus et caput cameli et per totum corpus habet stellas candidas multum rutilas et fulgentes cum colore reliquo sub albido. De pardalo [II, 2, 1, 17]

Cameleopardus, ut dicit Ysidorus, est animal simile pardo varium et albis maculis supersparsum. Sed collo est equo similis et habet pedes bubalinos. Solo vero capite similis est camelo. Nascitur autem in Ethyopia tantum. De cameleopardo [II, 2, 1, 39].

${ }^{55}$ Isidore de Séville, op. cit., 6, 35, p. 202-203.

${ }^{56}$ De uranoscopo [II, 2, 4, 30]. 
Comme il a été dit plus haut pour le bupreste, les noms grecs pouvaient poser de grands problèmes à Engelbert. Ainsi dans la notice du serpent cerastes, il affirme que son nom est composé du mot grec ceron ce qui s'écarte de l'étymologie donnée par Isidore ${ }^{57}$. Même si dans le processus de la transmission manuscrite du texte d'Isidore, des fautes ont pu s'être glissées, Engelbert aurait dû, s'il avait une connaissance de base du grec (ce qui reste à prouver), se rendre compte que le mot

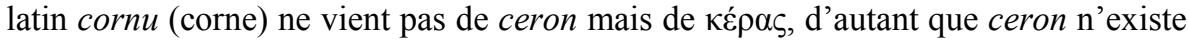
pas en grec.

L'influence d'Isidore sur le texte d'Engelbert est bien visible aussi pour le choix des noms des animaux. Engelbert adopte quasi toujours le nom de l'animal donné par Isidore ${ }^{58}$ sans ajouter le nom vulgaire. Sur ce point, il se distingue clairement de Thomas de Cantimpré (ca. 1201-ca. 1270) ou d'Albert le Grand ${ }^{59}$.

\section{Sources et citations dans le traité}

Le texte d'Isidore, comme celui d'Engelbert, est basé essentiellement sur une connaissance livresque ${ }^{60}$ et non sur une observation directe ${ }^{61}$. Pour copier et reproduire leurs informations, ils ont recours à la méthode d'excerptatio ou de compilation. Isidore ne cite pas systématiquement ses sources, contrairement à Engelbert $^{62}$, qui le fait régulièrement pour appuyer ses dires ${ }^{63}$. Ainsi dans tout le livre XII, Isidore ne fait que 58 citations d'auteurs, tandis qu'Engelbert cite au moins 706 fois un nom d'auteur. En tête de liste des auteurs les plus cités dans le Tractatus de naturis animalium figure Isidore (304), suivi par Solin. Les places 3 et 4 sont

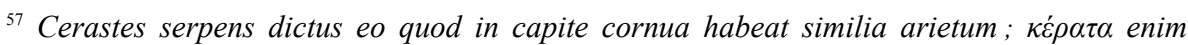
Graeci cornua uocant [...]. Isidore de Séville, op. cit., 4, 18, p. 147.

${ }^{58}$ Une exception est par exemple la scorpena [II, 2, 4, 12].

${ }^{59}$ Thomas de Cantimpré écrit par exemple : [...] raithe vel rais aut rochen, ut eas vulgus diversis linguis appellant [...] ou [...] sturio piscis est magnus, quem barbari storam vocant [...]. Thomas de Cantimpré, Liber de natura rerum, editio princeps secundum codices manuscriptos, partie 1 : texte, éd. H. Boese, Berlin-New York, 1973, VII, 68 et 70, p. 270.

${ }^{60}$ Des preuves qu'Isidore et Engelbert se basent surtout sur des textes et rarement sur l'observation personnelle sont par exemple l'emigraneus ("migraine»), pris chez les deux auteurs comme un ver dans la tête, et le poisson austral qui est considéré comme un véritable poisson. Voir Isidore de Séville, op. cit., p. 22. Voir les notices de tynea [II, 2, 3, 11] et de australi [II, 2, 4, 27].

${ }^{61}$ Mittelalterliche Naturlehre, das heisst verschriftete Naturkunde, geht bekanntlich nur in Ausnahmefällen auf eigene Naturbeobachtungen zurück; fast immer ist sie sekundäres Wissen, ausgehoben aus den einschlägigen Quellen. B. K. Vollmann, "Aal und Kili : zur mittelalterlichen Naturkunde», Aevum. Rassegna di scienze storiche, linguistiche $e$ filologiche, 73, 1999, p. 459

${ }^{62}$ Ce souci d'Engelbert de citer ses sources est manifeste dans la notice sur le chien : Canes, ut dicunt Ysidorus, Plinius, Solinus, Ambrosius, Aristoteles, sagacissima animalia sunt, [...]. De canibus [II, 2, 1, 11].

${ }^{63}$ Vincent de Beauvais a ce même souci de l'identification. B. Van den Abeele., «Vincent de Beauvais naturaliste : les sources des livres d'animaux du Speculum naturale », dans Lector et Compilator. Vincent de Beauvais, frère prêcheur, un intellectuel et son milieu au XIII siècle, S. Lusignan et M. Paulmier-Foucart (sous la dir.), Grâne, 1997, p. 135. 
occupées quasi ex aequo par Aristote (90) et Ambroise (87) devant de célèbres auteurs romains et des citations de l'Écriture sainte (17).

Tableau des auteurs les plus cités dans le Tractatus de naturis animalium ${ }^{64}$

\begin{tabular}{|c|c|c|c|c|c|c|c|c|c|}
\hline & Isidore & Solin & Aristote & Ambroise & Virgile & Bible & Lucain & $\begin{array}{l}\text { Experi- } \\
\text { mentator }\end{array}$ & $\begin{array}{l}\text { Pli } \\
\text { ne }\end{array}$ \\
\hline $\begin{array}{l}\text { Qui de } \\
\text { naturis } \\
\text { animalium }\end{array}$ & 1 & 3 & $1+1$ & 1 & 0 & 0 & 0 & 0 & 0 \\
\hline $\begin{array}{l}\text { Incipit ... de } \\
\text { natura } \\
\text { hominis }\end{array}$ & $14+1$ & $36+4$ & 24 & 0 & 1 & 5 & 0 & 0 & 0 \\
\hline $\begin{array}{l}\text { Incipit } \quad \text {... } \\
\text { naturis } \\
\text { animalium }\end{array}$ & 1 & 0 & 4 & 4 & 0 & 0 & 0 & 0 & 1 \\
\hline $\begin{array}{l}\text { De } \\
\text { gressibilibus }\end{array}$ & 87 & $78+6$ & $43+1$ & $23+4$ & $14+1$ & 5 & 0 & 10 & 8 \\
\hline $\begin{array}{l}\text { De } \\
\text { serpentibus }\end{array}$ & 38 & 12 & 1 & 2 & 1 & 3 & $9+(3)$ & 1 & 1 \\
\hline De vermibus & 7 & 0 & 4 & 0 & 0 & 1 & 0 & 0 & 0 \\
\hline De piscibus & 69 & $8+1$ & 3 & $26+1$ & $3+(1)$ & 1 & 0 & 1 & 2 \\
\hline De avibus & $77+1$ & $26+4$ & 6 & 24 & 14 & 2 & 3 & 2 & 0 \\
\hline $\begin{array}{l}\text { De minutis } \\
\text { volatilibus }\end{array}$ & 8 & 1 & 2 & 2 & 3 & 0 & 0 & 0 & 0 \\
\hline Total & $302+2$ & $164+15$ & $88+2$ & $82+5$ & $36+1+(1)$ & $17^{65}$ & $12+(3)$ & 14 & 12 \\
\hline
\end{tabular}

Le tableau montre que la fréquence d'un auteur peut varier sensiblement selon la catégorie. Ainsi dans la catégorie de gressibilibus, nous avons la configuration suivante : Isidore en tête (87), Solin en deuxième place (84), Aristote en troisième (44), Ambroise en quatrième (27) et Virgile en cinquième (15). Ce classement est identique à celui de l'œuvre complète. Par contre dans la catégorie des vers, ne figurent qu'Isidore (7), Aristote (4) et la Bible (1). La différence est très nette lorsqu'on compare le volet consacré à l'homme à celui des animaux. Ainsi dans la partie sur les animaux, Isidore figure en tête, alors que lorsqu'il s'agit de l'homme, il n'arrive qu'en troisième position (15), derrière Solin (40) et Aristote (24). Si l'on compare Isidore aux principaux autres auteurs regroupés on constate un phénomène semblable. Ainsi dans la catégorie des animaux marchant sur terre Isidore se situe en bas de l'échelle avec 87 citations contre 272 c'est-à-dire $32 \%$, alors que dans les cinq autres catégories Isidore occupe 48 à $59 \%$ de la place.

${ }^{64}$ Nous avons compté l'occurrence du nom d'auteur, mais aussi tous les idem et ibidem (chiffre derrière le signe d'addition et qui n'est pas entre parenthèses), ainsi que ses substituts comme poeta (chiffre entre parenthèses).

${ }^{65}$ Nous avons trouvé également cinq citations bibliques qui ne sont pas indiquées explicitement comme provenant de la Bible : de mulis [II, 2, 1, 13]; de ceto [II, 2, 4, 3]; de accipitre [II, 2, 5, 43] (2x) et de cyncendela [II, 2, 6, 7]. 
Il faut spécifier que parmi ces 58 citations d'auteurs faites par Isidore, 6 sont copiées directement de Servius ${ }^{66}$. Il s'agit de citations de «seconde main». Elles n'ont pas été prises directement à la source, mais puisées chez un auteur intermédiaire. Engelbert lui aussi fait largement usage de cette méthode de travail. Ainsi a-t-il repris des Étymologies 15 des 16 emprunts qu'Isidore a attribuées explicitement à Lucain ${ }^{67}$, trois des quatre de Cicéron ${ }^{68}$, un des deux d'Afranius, tous les emprunts d'Ovide (3), de $\operatorname{Macer}^{69}$ (2), de Dracontius (1), de Juvénal (1), de Martial (1), de Naevius (1), de Plaute (1) et de Virgile (1). Par contre il n'a pas copié les citations isidoriennes attribuées à Horace, Lucrèce, Perse et Suétone.

Engelbert a inséré aussi d'autres emprunts. Ainsi, il a ajouté 5 passages attribués à des auteurs, qui sont aussi cités par Isidore, mais dont les extraits ne figurent pas dans le livre XII des Étymologies: Horace (2), Cicéron (1) et Macer (1). À côté de la Bible et des auteurs principaux, qu'il cite tous au moins une dizaine de fois, Engelbert mentionne également les auteurs suivants qui ne sont pas du tout cités dans les livres XI et/ou XII : Grégoire le Grand (2), s. Jérôme (2), s. Augustin (1), Averroès (1), Avicenne (1), Boèce (1), Hippocrate (1), Jacques de Vitry (?) (1), Stace (1) et Varron (1). On voit qu'Engelbert ne cite pratiquement pas d'auteurs contemporains, ceci probablement pour des raisons d'autorité.

Notons qu'Engelbert manie les citations de façon différenciée. Ainsi les poètes comme Lucain ou Ovide sont repris le plus souvent verbatim et avec une phrase d'introduction ${ }^{70}$, alors que de façon générale il reformule en ses propres termes $^{71}$ les idées des prosateurs comme Pline ou Solin ${ }^{72}$. C'est dans la reformulation et l'enrichissement du texte d'Isidore que réside l'apport principal d'Engelbert.

\footnotetext{
${ }^{66}$ Isidore de Séville, op. cit., p. 13.

${ }^{67}$ Isidore a employé quatre fois l'expression poeta pour désigner Lucain. La seule citation de Lucain qu'Engelbert n'a pas reprise est la suivante: Cuius poeta sic meminit: Ossaque dissoluens cum corpore tabificus seps. Isidore de Séville, op. cit., 4, 31, p. 158-159.

${ }^{68}$ La seule qu'Engelbert n'a pas copiée est celle dans la notice du castor : De quibus Cicero in Scauriana: Redimunt se ea parte corporis, propter quod maxime expetuntur. Isidore de Séville, op. cit., 2, 21, p. 106-107.

${ }^{69}$ On voit clairement qu'Engelbert a copié ces passages du texte d'Isidore puisqu'il se trompe et attribue une des deux citations à Aemilius, alors que cette phrase provient du texte de Macer. Cette même erreur se trouve déjà dans le texte d'Isidore.

[...] sicut Aemilius ait: "Cygnus in auspiciis semper laetissimus ales; hunc optant nautae, quia se non mergit in undas. » Isidore de Séville, op. cit., 7, 19, p. 236-239.

Unde Emilius: "Cygnus in auspiciis semper letissimus ales : hunc optant naute, quia se non mergit in undas. » De cygno [II, 2, 5, 7].

${ }^{70}$ Est autem colore varius et pictus guttatim. Unde Ovidius ex re: Nomen habet variis stellatus corpore guttis. Aptum colori et cetera. De stellione [II, 2, 2, 28].

${ }^{71}$ Omne animal acutorum ungwium difficile parit, ut dicut Plinius. De generibus animalium [II, 1, 4].

${ }^{72}$ Cette même observation peut être faite pour l'œuvre d'Isidore. De quibus Lucretius: Scymnique leonum. et [...] sicut et Plinius in Naturali Historia dicit, leonem cum parda, aut pardum cum leaena concumbere [...]. Isidore de Séville, op. cit., 2, 6, p. 90-91 et 2, 11, p. 9497.
} 
À côté des emprunts explicites, le livre XII des Étymologies contient aussi une série d'emprunts non avoués. Ainsi Jacques André a repéré chez Isidore 293 emprunts non signalés en tant que tels et qui proviennent pour une grande partie des Pères de l'Église : Ambroise (27), Jérôme (23), Augustin (10) et Grégoire le Grand $(7)^{73}$.

Chez Engelbert, le nombre de citations inavouées n'est pas insignifiant non plus. Souvent Engelbert s'inspire d'Isidore sans le nommer expressément. Ainsi dans la catégorie des petits animaux volants, il reprend dans 17 notices individuelles les idées d'Isidore, mais ne le cite que 8 fois explicitement. Dans la catégorie des serpents presque $72 \%{ }^{74}$ du texte d'Engelbert est en relation étroite avec Isidore, dans les catégories des vers, poissons et petits animaux volants ce pourcentage varie entre 53 et 58 . La divergence entre la fréquence du nom d'Isidore cité et le volume textuel emprunté réellement n'est donc pas trop grande. Chez Engelbert, la relation entre le nombre d'emprunts non avoués et le nombre de citations semble néanmoins être moins importante que chez Isidore (293/58).

Toujours parmi les 293 emprunts non explicites dans le texte d'Isidore, 45 proviennent de Pline et 79 de Solin. Pour Pline, M. André a pu constater que plus de la moitié concernent les animaux aquatiques (28), alors que les bêtes sauvages et les quadrupèdes exotiques sont totalement exclus (sauf la lionne) ${ }^{75}$. Ce qui frappe dans les emprunts à Pline, c'est qu'Isidore a retenu avant tout les détails " merveilleux » et a omis les éléments descriptifs. De même, Engelbert a gardé la plupart de ces anecdotes touchant au fabuleux, sans se soucier des descriptions animales faites par Pline. Il se serait contenté des extraits fournis par des auteurs intermédiaires. À titre d'exemple, nous pouvons citer l'histoire de la grenouille (vivante ou morte) qui, une fois avalée par les chiens, les empêche d'aboyer ${ }^{76}$.

En ce qui concerne Solin, Isidore l'a utilisé principalement pour trois catégories : de bestiis, de serpentibus et de avibus. Lorsqu'Engelbert parle de Solin, il le cite explicitement. Dans la catégorie des animaux marchants, des serpents et des oiseaux, Solin est chaque fois le deuxième auteur le plus cité. Isidore et Engelbert ont eu recours à Solin surtout pour les animaux exotiques ${ }^{77}$ et les animaux «fabuleux $\left.{ }^{78}\right\rangle$.

Une source qui reste obscure est l'auteur appelé Experimentator. Isidore de Séville avait déjà signalé quelques propriétés d'animaux sous cette appellation et Engelbert a repris trois de ces passages ${ }^{79}$, mais Engelbert en a ajouté d'autres.

Il modo dell'utilizzazione [...] ora invece il dato di Solino viene modificato con più o meno evidenti rielaborazioni. F. Gasti, «I Collectanea di Solino come fonte del libro XI delle Etymologiae di Isidoro », Athenaeum, 66, 1988, p. 122.

${ }^{73}$ Isidore de Séville, op. cit., p. 15-16. Contrairement à Isidore, Engelbert cite explicitement le nom de s. Ambroise, s. Jérôme, s. Augustin et Grégoire le Grand.

${ }^{74}$ Dans cette catégorie 1.892 mots sur 2.617 sont inspirés d'Isidore.

${ }^{75}$ Isidore de Séville, op. cit., p. 17.

${ }^{76}$ Pline l'Ancien, Naturalis historia, XXXII, 140. Isidore de Séville, op. cit., 6, 59, p. 218 219. De ranis [II, 2, 4, 53].

${ }^{77}$ Voir de leone [II, 2, 1, 31] et de boa [II, 2, 2, 21].

${ }^{78}$ Voir de pegaso [II, 2, 5, 65].

${ }^{79}$ Isidore de Séville, op. cit., 4, 12, p. 142-143 ; 6, 46, p. 208-209 et 6, 59, p. 218-219. 
Certains de ces emprunts présentent des similarités avec des extraits du Liber de natura rerum de Thomas de Cantimpré, mais sans pour autant être tout à fait identiques ${ }^{80}$. Il ne semble pas s'agir de l'auteur inconnu que Thomas de Cantimpré cite fréquemment sous le nom d'Experimentator, et dont l'identité a donné lieu à des recherches récentes ${ }^{81}$.

\section{Méthodologie d'Engelbert}

Le prologue de l'œuvre, comme l'a remarqué M. Köhler, contient de précieuses indications sur la méthode de travail d'Engelbert. Ainsi Engelbert distingue trois approches différentes qui permettent l'acquisition de connaissances sur les animaux au sens large ${ }^{82}$.

La première approche est celle des philosophes (philosophi), dont Aristote est le champion. L'approche philosophique consiste à mettre en évidence les propriétés et différences physiologiques des animaux, à les expliquer et à en tirer des principes universels ${ }^{83}$.

La deuxième est celle des historiographes (historiographi) auxquels Engelbert associe les experimentatores. Parmi les historiographes, Engelbert cite Iacobus (Jacques de Vitry probablement) et surtout Solin. Le but des historiographes est d'impressionner le lecteur par des faits nouveaux et insolites, voire incroyables, touchant le monde des êtres sensibles ${ }^{84}$.

La troisième est celle des ethymologizatores, dont font partie Isidore de Séville et Ambroise de Milan. La finalité des ethymologizatores est de fournir et d'expliquer les noms des animaux et des espèces, de les décrire et d'y ajouter des

\footnotetext{
${ }^{80}$ Experimentator dicit quod gallus factus novem annorum ponit ovum, quod bufo acceptum fovet et exinde nascitur basyliscus. Voir de gallo [II, 2, 5, 37].

Gallus senescens in etate decrepita facit ovum ex se, unde basiliscus procreatur. Opinio quorundam est quod, postquam ediderit gallus ovum, id fovet coluber aut bufo. Thomas de Cantimpré, op. cit., V, 57, p. 205.

${ }^{81}$ Voir C. Hünemörder, «Die Lösung des Rätsels der sogenannten 3. Fassung (Thomas III) der naturkundlichen Enzyklopädie De natura rerum von Thomas von Cantimpré », Archives internationales d'histoire des sciences, 143, 1999, p. 252-268.

${ }^{82}$ T. W. Köhler, art. cit., p. 63-64.

${ }^{83}$ Quidam enim, ut philosophi, naturas et proprietates rerum per causas inquirentes, diversitates naturarum animalium tam in conplexione quam in conpositione parcium corporis eorum ex principiis neccessariis naturalis sciencie in quasdam utiles regulas ediderunt. Diversitates quoque omnium attencium et conswetudinum et morum, secundum quas animalia diversificantur, subtiliori et alciore speculacione in quasdam universales similiter regulas collegerunt et hoc modo de naturis animalium scripsit Aristoteles in libro suo, qui dicitur liber de animalibus, et plus in hystoria naturali idem facere conatus est. [Prologus].

${ }^{84}$ Alii vero non ut philosophi speculacionem de hiis habentes, sed ut hystoriographi et experimentatores nova et inusitata referre ad delectacionem legencium cupientes de naturis eorum varia scripserunt, id est de moribus et conswetis operacionibus animalium, non causam de singulis reddentes, sed quasi aliquid insolitum narrantes et hoc modo scripsit Solinus et Iacobus. Unde et Solinus librum suum, cuius tytulum postea mutavit, prius collectaneam rerum memorabilium nuncupavit. [Prologus].
} 
informations de nature diverse considérées comme importantes ${ }^{85}$. De ces trois catégories d'auteurs, seuls les ethymologizatores suivent un ordre et une structure précis dans l'arrangement des données sur la nature des animaux ${ }^{86}$.

Les informations venant des trois catégories sont acceptables à condition qu'elles soient approuvées par les autorités ${ }^{87}$. Voilà pourquoi Engelbert cite si souvent le nom des Anciens ${ }^{88}$. Dès le prologue, il prévient le lecteur, à la manière de Solin, qu'il ne doit pas s'attendre à des connaissances nouvelles en la matière puisque les Anciens ont déjà tout dit ${ }^{89}$.

Sans le préciser explicitement, Engelbert adopte et combine les trois approches de façon complémentaire ${ }^{90}$. Dans la partie sur l'homme, il prend souvent comme point de départ l'approche historiographique. Ainsi évoque-t-il d'abord une idée ou un passage des historiographes (Solin) qu'il critique ou explique ensuite à la manière des philosophes (Aristote) ${ }^{91}$. Dans la partie sur les animaux, Engelbert commence le plus souvent par les textes des ethymologizatores (Isidore) pour mettre à profit leur structure et complète ensuite la notice par d'autres sources ${ }^{92}$.

\footnotetext{
${ }^{85}$ Quidam vero non causas inquirentes rerum ut philosophi nec admirabilia referre intendentes ut experimentatores et hystoriographi, sed gratia vocum, id est nominum singularum rerum interpretandarum, de naturis ipsorum animalium aliqua tetigerunt, sicut Ysidorus in libro ethymologiarum et alii de ethymologiis nominum scribentes. Beatus quoque Ambrosius in libro Exameron de quorumdam animalium naturis quasi ex incidenti aliquid tangit; similiter et alii katholici tractatores. [Prologus].

${ }^{86}$ Diversum quoque ordinem tenuerunt. Nam philosophi et hystoriographi non continua narracione, sed sparsim hinc et inde de naturis, id est de conswetudinibus animalium dixerunt. Nominum vero ethymologizatores magis ordinem vocum quam rerum inspicientes secundum vocabulorum seriem de hiis scripserunt. [Prologus].

${ }^{87}$ [...], dum modo id, quod dicitur auctoritate antiquorum et testimonio, confirmetur. [Prologus].

${ }^{88}$ Ainsi dans la notice du serpent iaculus, Engelbert ne cite pas seulement le nom d'Isidore mais insiste pour ajouter celui de Solin, bien que l'information de Solin se trouve déjà comme telle dans le texte d'Isidore.

${ }^{89}$ Nam ut dicit Solinus, nichil novi afferre quasi a nobis ipsis possumus, cum diligencia antiquorum nil reliquerit intemptatum. [Prologus].

${ }^{90}$ T. W. Köhler, art. cit., p. 64.

${ }^{91}$ In utroque autem sexu, ut dicit Solinus, quando capilli incipiunt crescere, augetur magis dolor melioribus et in plenilunio plus dolent inpregnate et nati in plenilunio sunt debiliores et habent humidius cerebrum. Contrarium dicit Aristoteles quod noctes plenilunii calidiores sint et secundum hoc nati in plenilunio non haberent humidius cerebrum aliis. Quod menbrum primo formetur $[\mathrm{I}, 1,4]$.

${ }^{92}$ Locusta secundum Ysidorum dicitur ita ab eo quod longos habeat pedes sicut hasta et etiam duplex maritima et terrestris, quarum utramque Greci ab hasta hastaginem appellant. Hee, ut dicit Ambrosius, segetes devorant et suo morsu exurunt. Quibus avis, que Grece selence dicitur, insidiatur et devorat eas. De locustis [II, 2, 6, 10].
} 
L'approche philosophique est surtout réservée aux parties générales de chaque catégorie animalière ${ }^{93}$. Dans celles-ci, Engelbert explique le bien-fondé de la catégorie, la raison d'être de son nom et précise les propriétés communes de tous les animaux figurant dans ce collectif ${ }^{4}$. Si besoin en est, il développe les différentes sous-catégories et souligne les caractéristiques de ces animaux ${ }^{95}$.

\section{Raison d'être de son ouvre}

Les circonstances qui ont amené Engelbert d'Admont à écrire le Tractatus de naturis animalium ne sont pas transmises. Il n'y a nulle part d'indice prouvant qu'il aurait entamé ce travail suite à une demande d'un ami, comme dans le cas du Tractatus de fascinacione par exemple ${ }^{96}$.

Il semblerait donc qu'Engelbert ait écrit son œuvre de sa propre initiative. En tout cas, il est manifeste que le sujet de la connaissance de l'homme (de homine) l'a particulièrement intéressé, à en juger par les autres œuvres qui touchent à ce thème (Tractatus de fascinacione et Tractatus de causa longevitatis hominum ante diluvium, Tractatus de regimine principum, Tractatus metricus de consilio vivendi, ... $)^{97}$. Par contre, d'après le panorama de ses œuvres, Engelbert s'est intéressé moins à la faune qu'à l'homme. De toutes ses œuvres connues, à part cette œuvre-ci, seul le traité perdu Tractatus de quibusdam naturalibus aurait pu encore comporter d'importantes données « zoologiques ».

\footnotetext{
${ }^{93}$ Illius materie resultat virtus generativa anime in illa materia et hic est modus generacionis vermium, ut dicit Aristoteles XII methaphysice, quod idem facit virtus celi in materia putrefacta, quod patris virtus in semine. De vermibus [II, 2, 3, 1].

${ }^{94}$ Minuta volatilia apud auctores dicuntur duabus de causis vel quia minora sunt predictis omnibus et non ovant, sed coeunt ut apes, vel nascuntur ex putrefactis sine coitu ut scarabei et musce sicut in genere terrestrium. Formice et vermes minuta eadem ratione dicuntur, vel ideo quia quantitate corporis simpliciter minora sunt. De minutis volatilibus [II, 2, 6, 1].

${ }^{95}$ Gressibilium animalium alia sunt quadrupedia, alia bipedia, alia multipedia. [...] ; alia comedencia carnes, alia herbas et gramina. Omnia autem comedencia herbas habent colla longiora ceteris comedentibus carnes, ut dicit Ambrosius, et hec ex una parte tantum dentes habent. De gressibilibus [II, 2, 1, 1].

${ }^{96}$ Rogatus et interrogatus a quibusdam sociis et amicis ea que de hac materia videre potui et habere ab auctoribus inde dicta presenti tractatu duxi in unum [...]. G. B. Fowler, "Engelberti Admontensis Tractatus de fascinatione », Recherches de théologie ancienne et médiévale, 37, 1970, p. 195. T. W. Köhler, art. cit., p. 63.

${ }^{97}$ Engelbert a réparti ces œuvres en deux catégories: «In Philosophia Naturali» et «In Philosophya morali. » Or concernant ce point il y a une petite erreur dans l'article de Köhler. Il énumère 10 œuvres et continue: Mit Ausnahme des Speculum virtutum ordnet ENGELBERT alle diese anthropologisch relevanten Schriften den von ihm in der Epistola unterschiedenen Werksgruppen ,, de philosophia naturali “ oder ,de philosophia morali“ zu. L'œuvre Speculum virtutum moralium se trouve bel et bien dans la catégorie Philosophya morali, il s'agit de la troisième entrée : Item. Tractatum qui dicitur speculum virtutum. Voir $\mathrm{T}$. W. Köhler, art. cit., p. 63. J. Wichner, «Zwei Bücherverzeichnisse des 14. Jahrhunderts in der Admonter Stiftsbibliothek», Zentralblatt für Bibliothekswesen 1, complément 4, Leipzig, 1889 , p. 511 (=17).
} 


\section{Objectifs et limites de son oeuvre}

Les buts précis qu'Engelbert poursuit avec son œuvre sont la transmission de connaissances «scientifiques » et le divertissement du lecteur ${ }^{98}$. Pour ce qui est des données issues de la tradition littéraire des mirabilia, il les considère dans une certaine mesure aussi comme informations " scientifiques $^{99}$ »). Or, contrairement aux Pères de l'Eglise et à Hugues de Saint-Victor, il ne se prononce pas explicitement sur la finalité d'une telle connaissance en «zoologie». Ainsi ne rappelle-t-il pas au lecteur que la connaissance de la faune est un moyen pour l'homme de mieux appréhender la nature humaine ${ }^{100}$ et divine ou l'Écriture Sainte. M. Köhler est d'avis que pour Engelbert ces points étaient sous-entendus, il n'était donc pas nécessaire de les rappeler explicitement ${ }^{101}$.

Il n'y a pratiquement pas d'interprétation allégorique dans le Tractatus de naturis animalium. L'intention d'Engelbert n'était pas d'offrir aux prédicateurs un instrument de travail utile pour rendre leurs sermons plus vivants, contrairement à Thomas de Cantimpré ou Conrad de Mure (ca. 1210-1281) par exemple. Cette absence de moralisation et d'interprétation théologique confère au Tractatus de naturis animalium un caractère exceptionnel parmi les traités animaliers d'Europe centrale de la période allant de la fin du $13^{\mathrm{e}}$ jusqu'au $15^{\mathrm{e}}$ siècle. En effet, un très grand nombre de textes «naturalistes" de l'aire culturelle bavaro-autrichienne comportent cet aspect de lecture allégorique ${ }^{102}$. La moralisation animale joue un rôle

\footnotetext{
${ }_{98}$ Omnes vero hii simul non solum cognicion $<e>$ naturarum animalium profecerunt, sed etiam delectacioni, que est in rerum mirabilium auditu et percepcion $<i>$ non mediocriter consuluerunt ita ut huiusmodi legere non solum sit utile discentibus, sed etiam tediosis delectabile et iocundum, [...]. [Prologus]. Vu le grand nombre d'emprunts à Aristote, nous sommes d'avis qu'il ne faut pas privilégier le deuxième but, mais les considérer à pied d'égalité. Pour M. Ubl le deuxième aspect est plus caractéristique. K. Ubl, op. cit., p. 54.

${ }^{99}$ Sapiencia igitur est scientia multorum et mirabilium. [Compendium rhethorice Aristotilis], dans « Manuscript Admont 608 and Engelbert of Admont (c. 1250-1331) », éd. G. B. Fowler, Archives d'histoire littéraire et doctrinale du moyen âge, 52, 1977, p. 179. T. W. Köhler, art. cit., p. 65. S. Bazin-Tacchella, «Merveilles aquatiques dans les récits de voyage de l'époque médiévale », dans Dans l'eau, sous l'eau. Le monde aquatique au Moyen Âge, éd. D. JamesRaoul et C. Thomasset, Paris, 2002, p. 80.

${ }^{100}$ Ambroise de Milan, «Exameron», dans Sancti Ambrosii opera, pars I, éd. C. Schenkl, Prague-Vienne, 1897, VI, 2, 3, p. 205 (Corpus scriptorum ecclesiasticorum latinorum XXXII).

${ }^{101}$ T. W. Köhler, art. cit., p. 65.

${ }^{102}$ B. Van den Abeele, «Moralisierte Enzyklopädien in der Nachfolge von Bartholomäus Anglicus : Das ,Multifarium' in Wolfenbüttel und der ,Liber de exemplis et similitudinibus rerum' des Johannes de Sancto Geminiano", dans Die Enzyklopädie im Wandel vom Hochmittelalter bis zur frühen Neuzeit, C. Meier (sous la dir.), Munich, 2002, p. 296. B. Van den Abeele, «L'allégorie animale dans les encyclopédies latines du Moyen Âge », dans J. Berlioz et M.-A. Polo de Beaulieu (sous la dir.), L'animal exemplaire au Moyen Âge. $V^{e}$ $X V^{e}$ siècles, Rennes, 1999, p. 128-131. B. Van den Abeele, "Bestiaires encyclopédiques moralisés. Quelques succédanés de Thomas de Cantimpré et de Barthélémy l'Anglais », Reinardus, 7, 1994, p. 209-228.
} 
fondamental non seulement dans ces œuvres de l'espace culturel allemand, mais aussi dans des textes semblables en Italie ${ }^{103}$ et en France ${ }^{104}$.

Engelbert est conscient des limites de son œuvre. Son ambition n'est pas de décrire tous les animaux ou de mettre en évidence toutes leurs caractéristiques ${ }^{105}$ à la manière d'un Vincent de Beauvais ${ }^{106}$ ou Albert le Grand ${ }^{107}$. Contrairement à son Tractatus de fascinacione, l'œuvre ne cherche pas non plus à développer des connaissances nouvelles, mais elle veut rassembler ${ }^{108}$ et rendre compréhensible une partie importante du savoir des autorités, sous la forme d'un compendium. Sur ce point, il se rapproche d'Isidore de Séville. Engelbert préfère assembler un maximum de détails et d'anecdotes, plutôt que de répondre systématiquement à des questions théorico-philosophiques, comme il l'a fait dans son Tractatus de causis longevitatis hominum ante diluvium ${ }^{109}$.

Le public ciblé par cette œuvre est un milieu plus ou moins instruit ${ }^{110}$, mais non universitaire ${ }^{111}$. Il est possible qu'il ait écrit cette œuvre pour ses confrères ${ }^{112}$.

103 À retenir sont le Multifarium, écrit à Bologne vers 1326, le Liber de exemplis et similitudinibus rerum de Jean de San Gimignano, rédigé entre 1310 et 1327 et le Tractatus septiformis de moralitatibus rerum de Marc d'Orvieto, achevé à la fin du XIII ${ }^{\mathrm{e}}$ siècle.

${ }^{104}$ Nous pensons surtout au Reductorium morale de Pierre Bersuire (ca. 1290-1362).

${ }^{105}$ Ceteras regulas generales require in libro Aristotelis de animalibus. De specialibus enim solum est hic intencio. De generibus animalium [II, 1, 4]. M. Köhler est aussi d'avis que l'oeuvre parait inachevée et moins uniformisée par rapport à d'autres d'Engelbert. T. W. Köhler, art. cit., p. 62 et 72.

106 M. I. Gerhardt, «Zoologie médiévale: préoccupations et procédés », Miscellanea mediaevalia, 7, 1970, p. 240.

${ }^{107} \mathrm{M}$. Ubl évoque deux facteurs qui pourraient expliquer cette modestie d'Engelbert. Ce dernier s'est rendu compte qu'avec le nombre limité de livres à sa disposition à Admont et l'absence d'une culture de disputatio, il serait incapable de concevoir une œuvre systématique d'une ampleur comparable à celle d'un De animalibus. Cette remarque serait aussi valable pour ses autres œuvres de philosophie naturelle. C'est pourquoi Engelbert aurait préféré examiner des points « secondaires » et très spécifiques (l'âge des hommes avant le Déluge par exemple), plutôt que de se pencher sur les grandes questions de la philosophie naturelle. K. Ubl, op. cit., p. 54 et 224.

${ }^{108}$ Dans le domaine encyclopédique, la compilation était plus estimée que l'invention personnelle [...]. M. I. Gerhardt, art. cit., p. 241.

${ }^{109}$ T. W. Köhler, Grundlagen des philosophisch anthropologischen Diskurses im dreizehnten Jahrhundert. Die Erkenntnisbemühung um den Menschen im zeitgenössischen Verständnis, Brill, 2000, p. 104-105.

${ }^{110}$ Primo ergo de domesticis, que ex usu nociora sunt, dicamus propter legentis solacium magis quam ad profectum discencium. De gressibilibus [II, 2, 1, 1].

${ }^{111}$ T. W. Köhler, op. cit., p. 103.

112 Die schnelle Aufeinanderfolge der naturkundlichen Enzyklopädien spricht für ein ausserordentlich lebhaftes Interesse des monastischen Lesepublikums im 13. Jahrhundert an ihnen. C. Hünemörder, «Die Bedeutung und die Arbeitsweise des Thomas von Cantimpré und sein Beitrag zur Naturkunde des Mittelalters ", Medizinhistorisches Journal, 3, 1968, p. 355 . 
Vieux de 600 ans, le texte des Étymologies n'avait rien perdu de sa popularité au $13^{\mathrm{e}}$ siècle. Engelbert a utilisé les livres XI et XII comme source principale de son Tractatus de naturis animalium, bien que des œuvres plus développées et plus récentes sur la nature des animaux lui aient été accessibles. Il n'a pas seulement gardé la structure du texte isidorien, mais il a également conservé le même type d'information. Contrairement au goût de l'époque il n'a pas inséré de moralisation ou d'allégories. Peut-être ce choix explique-t-il le peu de succès de son traité, resté confidentiel?

Max Schmitz 